\title{
PROPIEDADES DE TABLEROS DE PARTÍCULAS DE PinUs radiata D.Don CON ASERRÍN DE LA MISMA ESPECIE
}

\author{
ANDRÉS BERTERRECHE ${ }^{(\bullet)}$ HERNÁN POBLETE ${ }^{(\cdots)}$ y PETER NIEMZ ${ }^{(\cdots *)}$
}

(*) Ing. Forestal. Universidad Católica del Uruguay. CP 11600, Montevideo Uruguay. FAX (598-2) 470323

(*) Dr. Ing. Forestal. U. Austral de Chile. Fac.Cs.Forestales. Cas.567. Valdivia-Chile. FAX (063) 221227

(*) Dr. Ing. Forestal. U. Austral de Chile. Fac.Cs.Forestales. Cas.567. Valdivia-Chile. FAX (063)221227

\section{RESUMEN}

Se estudió el efecto del contenido de aserrín y la densidad en las propiedades: Hinchamiento, Dureza Janka y Flexión Dinámica (impacto), Flexión Estática (MOR y MOE) y Tracción Perpendicular en tableros de particulas de Pinus radiata D. Don.

Las caracteristicas de los tableros fueron: Capas: 3 (20\%-40\%-20\%); Espesor: 16 mm; Contenido de aserrin: $0 \%$ a $100 \%$; Densidad: $570 \mathrm{~kg} / \mathrm{m}^{3}$ y $660 \mathrm{~kg} / \mathrm{m}^{3}$; Adhesivo: Ureaformaldehido.

Todas las propiedades mecánicas se ven afectadas positivamente por incrementos de la densidad, mientras que el porcentaje de aserrin sólo lo hace en relación a la propiedad de Tracción. Flexión Estática (MOR y MOE). Dureza Janka y Flexión Dinámica (Impacto) se correlacionan negativanente con un contenido de aserrin más alto.

Palabras Clave: Tableros de Particulas, Pinus radiata

\section{ABSTRACT}

The effect of saudust content and density on properties such as thickness swelling, Janka hardness, dynamic bending (impact), MOR, MOE, and IB were determinated in particle boards of Pinus radiata D. Don

Board characteristics were: Lavers: 3 (20\%-40\%-20\%); Thickness: 16 mm; Sawdust comtent: $0 \%$ to $100 \%$ : Board density: $570 \mathrm{~kg} / \mathrm{m}^{3}$ and $660 \mathrm{~kg} / \mathrm{m}^{3}$; Adhesive: Ureaformaldehyd.

All mechanical properties were positively affected by increasing density, while sawdust content was only positively related to Intenal Bond. Bending strenght (MOR and MOE), Janka hardness, and dimanic flexure (Impact) were negatively correlated with higher sawdust content. 


\section{INTRODUCCIÓN.}

La inclusión de desechos en la fabricación de tableros de partículas ha sido objeto de numerosos estudios (Gillwald, 1965: Gerischer. 1977).

En la fabricación de tableros de particulas ha aumentado significativamente la inclusión de finos y aserrín. Baste señalar que en el caso de Chile las plantas más modernas se abastecen en forma importante con este material.

Al incorporar aserrin al tablero en reemplazo de las partículas tradicionales se provocan cambios en prácticamente todas las propiedades mecánicas (Jensen, 1978; Poblete. 1979).

La inclusión de partículas mas pequeñas pucde provocar un aumento de la resistencia a la tracción y una disminución de la flexión estática (Post. 1958. Poblete. 1985).

En relación a las propiedades fisicas. no existe claridad en cuanto al efecto de agregar un material de menores dimensiones. Existen resultados contradictorios al respecto (Berterreche. 1994).

Los trabajos acerca de las propiedades físico-mecánicas de tableros fabricados con distintos contenidos de aserrín de Pinus radiata creciendo en Chile son escasos.

Existen además algunas propiedades especialmente importantes cuyo comportamiento aún no ha sido estudiado en profundidad y no se ha llegado a una aclaración total en relación a cómo son afectadas por la inclusión de aserrín. Entre ellas se pueden citar flexión dinámica y dureza. las que tienen importancia desde el punto de vista de las posibilidades de uso de los tableros.

\section{OBJETIVOS.}

Los objetivos del presente trabajo son:

- Determinar el comportamiento de tableros de partículas con dos niveles de densidad. frente a la inclusión de aserrin de Pinus radiata D.Don. en las capas externas y media.

- Determinar cuál es cl porcentaje máximo admisible. desde el punto de vista técnico. de aserrin en tableros de particulas de tres capas. 


\section{MATERIALES Y MÉTODO.}

\section{Madera}

Como material de ensayo se utilizó aserrín proveniente de pino insigne (Pinus radiata D. Don) la X Región producido en el corte con sierra sin fin (huincha) (Gentileza de la empresa Ascrraderos ARAGON).

Se emplearon además partículas de madera de la misma especie producidas a partir de astillas en un viruteador de anillos con cuchillos del tipo PALLMANN.

\section{Adhesivo}

El adhesivo utilizado fue ureaformaldchido. aplicado en solución con $50 \%$ de sólidos. con la adición de un catalizador $\left(\mathrm{NH}_{4} \mathrm{Cl}\right)$ en solución al $20 \%$ y en una proporción del $0.5 \%$.

\section{Método}

\section{Análisis del Aserrin y de las Particulas}

En la caracterización de la geometría y tamaño de ambas fracciones se trabajó de acuerdo con la metodología propuesta por Poblete (1979). Para ello se clasificaron muestras. emplcándose cinco tamices $(4.0-2.0-1.0-0.5 \mathrm{~mm})$.

La determinación de la densidad se efectuó mediante una relación entre el peso del material en estado anhidro y el volumen medido en estado saturado. El volumen se midió por desplazamiento de agua.

La determinación del valor $\mathrm{pH}$ se realizó en suspensiones de agua destilada ! madera. Para cllo se empleó el material de la fracción más fina obtenida del análisis de tamaño $(<0.5 \mathrm{~mm}$ ). Las suspensiones se efectuaron mezclando $10 \mathrm{~g}$ de material en 100 $\mathrm{ml}$ de agua destilada y las mediciones se realizaron después de + horas de agitación. determinándose $\mathrm{cl}$ valor del $\mathrm{pH}$ mediante un $\mathrm{pH}-$ metro.

Fabricación de los Tableros

Tanto las partículas como cl aserrin fueron secados hasta un contenido de humedad aproximado de $4 \%$. El aserrin fue tamizado para separarlo en tres fracciones. De este modo la fracción más gruesa se dispuso para la capa media. la media para la capa extcrna y la más fina (polvo) se desechó. 
Los tableros tuvieron las siguientes características:

Capas : 3 ( $40 \%$ capas externas; $60 \%$ capa media).

Proporciones de aserrin : 0 a $100 \%$. en rangos de $20 \%$. Estas proporciones fueron del mismo tenor para las caras y para la capa media.

Densidad : $570 \mathrm{~kg} / \mathrm{m}^{3}$ y $660 \mathrm{~kg} / \mathrm{m}^{3}$.

Espesor $16 \mathrm{~mm}$.

Encolado : $10 \%$ capas externas; $7 \%$ capa media. Promedio: $8,2 \%$.

En total se realizaron 12 tratamientos (seis contenidos de aserrín para cada densidad) con cuatro repeticiones. lo que dio un total de 48 tableros.

En el prensado se consideró una presión máxima de $3.5 \mathrm{~N}$. un tiempo total de 6 min y una temperatura de $160^{\circ} \mathrm{C}$.

Después de su fabricación. los tableros fueron climatizados según la norma DIN 50014

Determinación de las Propiedades de los Tableros

Los ensayos físicos y mecánicos se realizaron según los procedimientos especificados en normas DIN y ASTM. La evaluación de los resultados se efectuó teniendo en consideración las exigencias de la norma DIN 68763.

Las normas empleadas se enumeran a continuación:

Densidad y humedad : : DIN 52.361.

Hinchamiento : DIN 52364 .

Absorción de agua $\quad:$ ASTM 1037.

Flexión estática (MOR y MOE) : DIN 52362.

Tracción perpendicular al plano : DIN 52365 .

Además de los ensayos señalados. se efectuaron determinaciones en las que se emplearon metodologías no normalizadas con adaptaciones de otras normas. Estos métodos se describen a continuación:

Perfil de densidad

La determinación del perfil de densidad vertical se rcalizó mediante radiaciones Gamma (Gentileza de la empresa FIBRANOVA S.A.) Esta propiedad se evaluó sobre probetas de $50 * 50 \mathrm{~mm}$. 
Flexión dinámica (Impacto) :

Dureza Janka
Para el caso de flexión dinámica se utilizó una modificación de la norma DIN 52189 , norma para madera sólida, con una distancia entre los puntos de apoyo de $140 \mathrm{~mm}$ y utilizando para el ensayo dos probetas encoladas sumando un ancho de $32 \mathrm{~mm}$.

Para el ensayo de dureza Janka se procedió a partir de una modificación de la norma chilena NCh $978 . c 85$ con probetas de $16 * 50 * 100 \mathrm{~mm}$.

\section{RESULTADOS Y DISCUSION}

\section{Granulometria del Material Usado}

El aserrin utilizado presentó una forma cercana a la cúbica, con dimensiones en longitud y espesor similares. Esto concuerda con lo descrito por otros autores (Klauditz y Buro. 1962: Poblete. 1979). Visualmente se pudo constatar una mayor cantidad de corteza en el aserrin que en las partículas.

Los resultados del estudio de granulometría del aserrin y de las partículas se presentan en el Cuadro $\mathrm{N}^{\circ} 1$. Las fracciones finas fueron destinadas a las capas externas $\mathrm{y}$ las fracciones gruesas a la capa media del tablero.

\section{Cuadro $N^{\circ} 1$. \\ COEFICIFNTE DE ESBELTEZ}

\begin{tabular}{|lc|}
\hline Tipo de Fracción & Coeficiente de Esbeltez \\
\hline Aserrin sin Clasificación & 6.30 \\
\hline Aserrin con clasificación Fracción Fina & 5.31 \\
\hline Aserrin con clasificación Fracción Gruesa & 7,17 \\
\hline Particulas Tradicionales Fracción Fina & 18.33 \\
\hline Particulas Tradicionales Fracción Fina & 29.74 \\
\hline
\end{tabular}

En el aserrín sin clasificación se observó que el $85 \%$ del material fue retenido en mallas de 0.5 a $2.0 \mathrm{~mm}$. con un largo promedio de $2.9 \mathrm{~mm}$ y un espesor promedio de $0.46 \mathrm{~mm}$. El coeficiente de esbeltez. registrado $(7.1)$ se encuentra dentro de lo esperado para este tipo de partículas.

La fracción fina del aserrín clasificado concentró un $80 \%$ del material en mallas de 0.5 a $1.0 \mathrm{~mm}$. El largo promedio fue $2.6 \mathrm{~mm}$ y el espesor promedio $0,49 \mathrm{~mm}$. En el tamizado de la fracción gruesa del aserrin clasificado se retuvo más del $90 \%$ entre mallas de 0.5 y $2.0 \mathrm{~mm}$. Su largo promedio fue $3.3 \mathrm{~mm}$ y su espesor promedio fue 
$0,66 \mathrm{~mm}$. El coeficiente de esbeltez de ambas fracciones fue menor al del material sin clasificar. Esto se debe a la eliminación del material de mayor tamaño.

El largo promedio de la fracción gruesa de las partículas tradicionales fue $11,6 \mathrm{~mm}$ y su espesor promedio fue $0,39 \mathrm{~mm}$. El coeficiente de esbeltez ponderado correspondió a lo usado tradicionalmente por la industria. En la fracción fina de las partículas tradicionales, al igual que en el aserrin, se produjo una mayor concentración entre las mallas de 0.5 a $2,0 \mathrm{~mm}$. Las partículas fueron más largas que las de aserrín, $5,5 \mathrm{~mm}, \mathrm{y}$ el espesor fue menor, $0,30 \mathrm{~mm}$.

Las diferencias en la geometría del aserrin y de las partículas afectan directamente a las propiedades mecánicas de los tableros. En términos generales con aumentos del coeficiente de esbeltez se provocan aumentos de la flexión y disminuciones de la tracción (Post, 1958; Kusian. 1966; Bhagwat. 1971; Poblete 1985).

\section{Densidad y Valor de pH del Aserrín}

La densidad promedio de las partículas de aserrín alcanzó a $412 \mathrm{~kg} / \mathrm{m}^{3}$, con valores extremos de 385 y $435 \mathrm{~kg} / \mathrm{m}^{3}$. Estos valores pueden considerarse dentro de las densidades normales que presenta el pino insigne.

El valor de $\mathrm{pH}$ obtenido fue de 4.40. Este se encuentra dentro de los límites que se mencionan en la litcratura consultada (Pobletc y Sánchez. 1991).

\section{Propiedades Físicas de los Tableros}

\section{Perfil de Densidad}

Los resultados de Perfil de Densidad fueron los esperados. presentando los diagramas respectivos la forma típica para este tipo de producto. En la Figura $\mathrm{N}^{\circ}$ l se presentan ejemplos del diagrama de tableros con $570 \mathrm{~kg} / \mathrm{m}^{3}$ y $660 \mathrm{~kg} / \mathrm{m}^{3}$.

En todos los tratamientos se obtuvo una densidad máxima situada entre 1,0 y 2,5 $\mathrm{mm}$ debajo de cada superficie del tablero y una depresión de esta variable en el centro del mismo. 

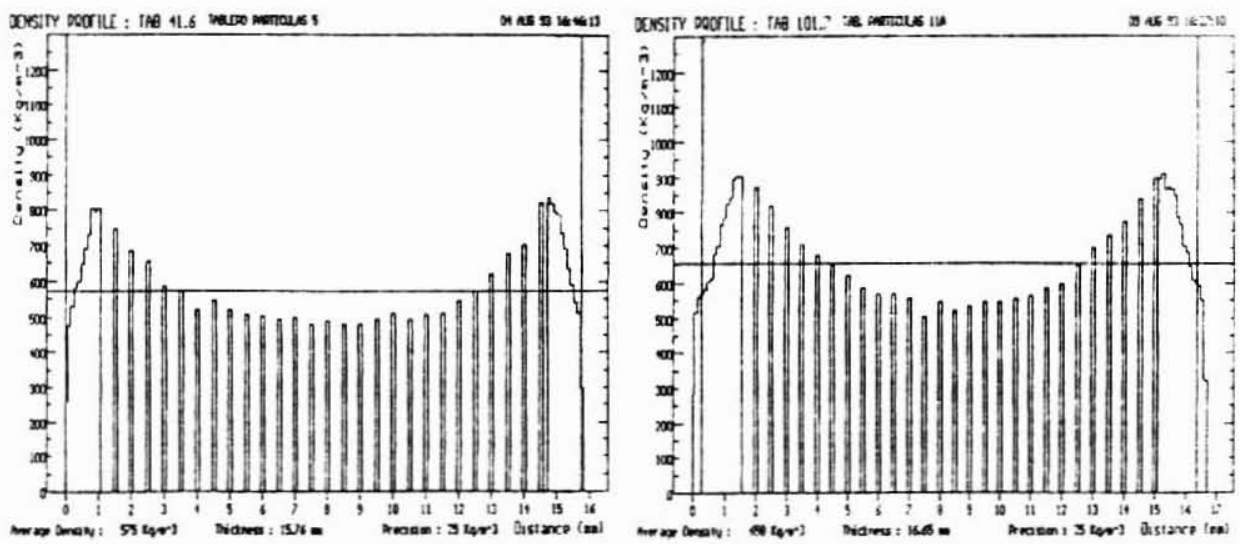

\begin{tabular}{|c|c|c|c|}
\hline \multicolumn{4}{|c|}{ SAMPLE PARAMETERS } \\
\hline \multirow{2}{*}{\multicolumn{4}{|c|}{$\begin{array}{l}\text { Sample ID \& DOS file : TAB } 41.6 \\
\text { Desc: TABLERO PARTICULAS } 5\end{array}$}} \\
\hline & & & \\
\hline Date & & & 04 AUG 93 \\
\hline & & : & $16: 46: 13$ \\
\hline ol der & & : & \\
\hline cision & $\left(\mathrm{Kg} / \mathrm{m}^{\wedge} 3\right)$ & : & \\
\hline Sample Thickness & $(\mathrm{mm})$ & : & 15.76 \\
\hline nal Th & $(\mathrm{mm})$ & : & \\
\hline eak Density Left & $\left(\mathrm{Kg} / \mathrm{m}^{\wedge} 3\right)$ & : & \\
\hline Density Right. & $\left(\mathrm{Kg} / \mathrm{m}^{\wedge} 3\right)$ & : & 83 \\
\hline Uur face Density Left & $\left(\mathrm{Kg} / \mathrm{m}^{\wedge} 3\right)$ & : & 47 \\
\hline Surface Density Right & $(\mathrm{Kg} / \mathrm{m} \wedge 3)$ & : & 513 \\
\hline Surtace Density & $\left(\mathrm{Kg} / \mathrm{m}^{\wedge} 3\right)$ & : & 49 \\
\hline ore Density & $(\mathrm{Kg} / \mathrm{m} \sim 3)$ & : & 48 \\
\hline$x$-Press Density & $(\mathrm{Kg} / \mathrm{m} \cap 3)$ & $:$ & 57 \\
\hline ander Density & $(\mathrm{Kg} / \mathrm{m}-3)$ & : & 57 \\
\hline ore to Ex-Sander Rat & & : & 0.8 \\
\hline
\end{tabular}

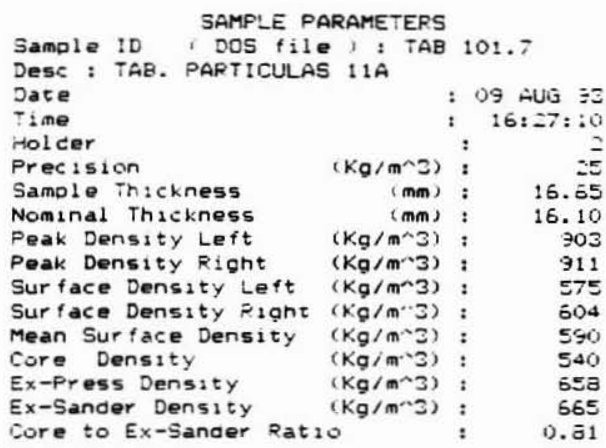

Figura $N^{\circ}$ 1. DIAGRAMA DEL PERFIL DE DENSIDAD EN TABLEROS DE DE AMBOS NIVELES DE DENSIDAD

El perfil de densidad no fue afectado por la cantidad de aserrin incluida. El análisis estadistico de la relación densidad maxima/densidad capa media, que está correlacionada positivamente con las resistencias mecánicas, tampoco reveló la presencia de diferencias significativas. Se pudo establecer además que no existen diferencias importantes entre las caras de un mismo tablero. 


\section{Humedad}

La humedad de los tableros al momento de los ensayos, climatizados según DIN 52 361 , varió entre $7,98 \%$ y $8,50 \%$. Las humedades promedio por tratamiento estuvieron dentro de lo requerido por la norma, entre 5 y $11 \%$ de contenido de humedad en los tableros.

\section{Hinchamiento a las 2 y 24 Horas}

En el tratamiento de dos horas los tableros excedieron el limite exigido por la norma, $8 \%$ (Cuadro $\mathrm{N}^{\circ} 3$ ). El análisis de varianza demostró que existen diferencias significativas, a un nivel de significación del $5 \%$. entre las densidades estudiadas. En el tratamiento de dos horas se determinó que al aumentar la densidad disminuye el hinchamiento.

El que en ninguno de los tratamientos se cumplió con la norma no es importante ya que bastaria con agregar un hidrófobo en la fabricación del tablero para mejorar esta propiedad. La decisión de no incorporar esta sustancia en el ensayo se debió a que la ausencia de hidrófobo permite visualizar mejor las diferencias entre los tratamientos.

La norma DIN determina un hinchamiento máximo de $16 \%$ a 24 horas. Solo los tratamientos con 0.40 y $80 \%$ de aserrin y densidad $570 \mathrm{~kg} / \mathrm{m}^{3}$ cumplieron con el requisito. Todos los tableros de densidad $660 \mathrm{~kg} / \mathrm{m}^{3}$ superaron la exigencia de la norma (Cuadro $\mathrm{N}^{\circ} 3$ ). El análisis de varianza determinó que existen diferencias significativas. Tanto el porcentaje de aserrín como la densidad determinaron dichas diferencias.

En el hinchamiento a 24 horas la densidad fue la variable determinante en esta propicdad: el contenido de aserrín la afectó en menor proporción. 


\section{Cuadro $\mathrm{N}^{\circ} 3$}

HINCHAMIENTO DE LOS TABLEROS A 2 Y 24 HORAS.

\begin{tabular}{|c|c|c|c|c|c|}
\hline \multirow{2}{*}{\multicolumn{2}{|c|}{$\begin{array}{ll} & \text { Aserrin } \\
\end{array}$}} & \multicolumn{2}{|c|}{ Hinchamiento $2 \mathrm{~h}(\%)$} & \multicolumn{2}{|c|}{ Hinchamiento $24 \mathrm{~h}(\%)$} \\
\hline & & Den. 570 & Den. 660 & Den. 570 & Den. 660 \\
\hline 0 & $\begin{array}{l}\text { Media } \\
\text { Máxima } \\
\text { Minima } \\
\text { D. E: }\end{array}$ & $\begin{array}{r}11,28 \\
12,58 \\
9,38 \\
0,93\end{array}$ & $\begin{array}{r}10,69 \\
12,50 \\
8,70 \\
1,20\end{array}$ & $\begin{array}{r}14,81 \\
16,77 \\
12,35 \\
1,22\end{array}$ & $\begin{array}{r}16,13 \\
20,25 \\
14,20 \\
1,57\end{array}$ \\
\hline 20 & $\begin{array}{l}\text { Media } \\
\text { Máxima } \\
\text { Minima } \\
\text { D. E: }\end{array}$ & $\begin{array}{r}13,25 \\
14,72 \\
10,69 \\
1,24\end{array}$ & $\begin{array}{r}11,77 \\
13,58 \\
9,43 \\
1,16\end{array}$ & $\begin{array}{r}17,30 \\
20,50 \\
14,91 \\
1,75\end{array}$ & $\begin{array}{r}17,14 \\
19,50 \\
14,47 \\
1,40\end{array}$ \\
\hline 40 & $\begin{array}{l}\text { Media } \\
\text { Maxima } \\
\text { Minima } \\
\text { D. E: }\end{array}$ & $\begin{array}{r}11,29 \\
13,21 \\
10,00 \\
0.92\end{array}$ & $\begin{array}{r}11,06 \\
13,04 \\
10.00 \\
0.88\end{array}$ & $\begin{array}{r}15,20 \\
16,88 \\
13,58 \\
1,12\end{array}$ & $\begin{array}{r}16,54 \\
18,87 \\
15,00 \\
1,24\end{array}$ \\
\hline 60 & $\begin{array}{l}\text { Media } \\
\text { Máxima } \\
\text { Minima } \\
\text { D. E: }\end{array}$ & $\begin{array}{r}12,72 \\
15.00 \\
11,25 \\
1.24\end{array}$ & $\begin{array}{r}11,47 \\
13.13 \\
9,88 \\
1.04\end{array}$ & $\begin{array}{r}16,41 \\
18,52 \\
14,82 \\
1,16\end{array}$ & $\begin{array}{r}18,22 \\
20,63 \\
15,43 \\
1,49\end{array}$ \\
\hline 80 & $\begin{array}{l}\text { Media } \\
\text { Maxima } \\
\text { Minima } \\
\text { D. E: }\end{array}$ & $\begin{array}{r}10,94 \\
13,67 \\
8.13 \\
1.44 \\
\end{array}$ & $\begin{array}{r}10.51 \\
12.88 \\
8.08 \\
1.58\end{array}$ & $\begin{array}{r}15,06 \\
18,63 \\
11,25 \\
2.12\end{array}$ & $\begin{array}{r}17,87 \\
21,38 \\
14,91 \\
1,59\end{array}$ \\
\hline 100 & $\begin{array}{l}\text { Media } \\
\text { Máxima } \\
\text { Minima } \\
\text { D. E: }\end{array}$ & $\begin{array}{r}12,96 \\
14.63 \\
11.66 \\
0.94\end{array}$ & $\begin{array}{r}10,83 \\
15,43 \\
6,59 \\
2,16\end{array}$ & $\begin{array}{r}17,14 \\
18,90 \\
13.58 \\
1,34\end{array}$ & $\begin{array}{r}17,54 \\
19,76 \\
14,79 \\
1,76\end{array}$ \\
\hline
\end{tabular}

D.E. $=$ Desviación Estandar

El efecto del aserrin se debe a que estas partículas tienen un espesor mayor y sufren una deformación en el momento del prensado. la que se libera cuando el tratamiento de inmersión es prolongado. Este efecto concuerda con los resultados de varios autores (Brumbaugh. 1960; Post. 1958; Jorgensen y Odell, 1961).

Lo anterior se corroboró por el comportamiento de la variable densidad. En el caso de los tableros de menor densidad el material se encuentra menos compactado y por lo tanto sus partículas sufren una menor deformación. Cuando ellas recuperan su forma original el cambio del espesor es menor. No se descarta la posibilidad que este efecto retardado del agua esté también regulado por su efecto sobre el adhesivo: A medida que éste se degrada la acción del agua sobre la madera intensificaría el hinchamiento.

\section{Absorción de Agua a las 2 y 24 Horas}

Los resultados obtenidos para la absorción a las 2 y 24 horas se presentan en el Cuadro $\mathrm{N}^{\circ} 4$. Para el caso de la absorción de agua no cxisten normas DIN o ASTM que fijen un limite máximo. 


\section{Cuadro $\mathrm{N}^{\circ} 4$}

\section{ABSORCION DE AGUA}

\begin{tabular}{|c|c|c|c|c|c|}
\hline \multirow{2}{*}{\multicolumn{2}{|c|}{ Aserrin }} & \multicolumn{2}{|c|}{ Absorción $2 \mathrm{~h}(\%)$} & \multicolumn{2}{|c|}{ Absorción $24 \mathrm{~h}(\%)$} \\
\hline & & Den. 570 & Den. 660 & Den. 570 & Den. 660 \\
\hline \multirow{4}{*}{0} & Media & 82,48 & 61,80 & 101,79 & 80,74 \\
\hline & Maxima & 95.79 & 76,38 & 118,34 & 95,50 \\
\hline & Minima & 57,85 & 38.59 & 74,96 & 66,62 \\
\hline & D, E: & 10,27 & 11.71 & 11,81 & 9,09 \\
\hline \multirow{4}{*}{20} & Media & 88,30 & 62,42 & 108,12 & 81,71 \\
\hline & Máxima & 110.66 & 78,88 & 127,71 & 92,90 \\
\hline & Minima & 66.77 & 53.69 & 85,56 & 71,31 \\
\hline & D, F: & 13.44 & 9.13 & 12.42 & 7.43 \\
\hline \multirow{4}{*}{40} & Media & 72.82 & 58,76 & 97,11 & 82,73 \\
\hline & Máxima & 88.00 & 70.44 & 117,44 & 96,45 \\
\hline & Minima & 58.08 & 46.55 & 83,63 & 69.23 \\
\hline & D. E: & 9.34 & 7.44 & 9.23 & 7.86 \\
\hline \multirow{4}{*}{60} & Media & 90,19 & 57,25 & 106,57 & 81,31 \\
\hline & Máxima & 118,20 & 75,88 & 125.44 & 98,07 \\
\hline & Minima & 73.94 & 47.56 & 91,95 & 69,58 \\
\hline & D. F: & 12.05 & 9.45 & 8.60 & 8.09 \\
\hline \multirow{4}{*}{80} & Media & 81,13 & 57.25 & 106,57 & 84,70 \\
\hline & Maxima & 96.60 & 69,87 & 134.89 & 98,38 \\
\hline & Minima & 65.61 & 42.51 & 91,86 & 78,02 \\
\hline & D. E: & 10.96 & 9.42 & 11.08 & 6,34 \\
\hline \multirow{4}{*}{100} & Media & 89.10 & 63.71 & 111,62 & 92,51 \\
\hline & Máxima & 108.64 & 82.56 & 128.27 & 112,18 \\
\hline & Minima & 72.99 & 44.31 & 99,05 & 80,79 \\
\hline & D. E: & 11.29 & 12.88 & 8,13 & 8,79 \\
\hline
\end{tabular}

D.E. $=$ Desviación Estándar

Al realizar el análisis de varianza para el tratamiento a 2 horas, se determinó que existen diferencias significativas y que estas se deben a la densidad. El aumento de ésta determina una disminución de la absorción. Esto puede explicarse por la presencia de una menor porosidad del material como consecuencia de una mayor compresión.

En el ensayo de inmersión durante 24 horas se determinaron diferencias significativas entre los tratamientos. En este caso apareció como predictor de dicha variación el porcentaje de aserrín pero con una menor relevancia que la densidad. El aserrín actuó aumentando la absorción de agua. Esto posiblemente se deba a la geometria del aserrín con una mayor superficic especifica y. por consecuencia, una menor cantidad de adhesivo disponible por superficie. Lo anterior determinaria una mayor facilidad del material para absorber agua.

Los resultados de la absorción de agua reafirmaron lo detcrminado en los ensayos de hinchamiento a las 2 y 24 horas: el agua necesitó actuar por un tiempo prolongado para que el efecto del aserrin sea detectable. 


\section{Propiedades Mecánicas de los Tableros}

Tracción Perpendicular al Plano

Esta propiedad mecánica es un indicador de la calidad de la unión entre las partículas de la capa media (Gatchell et al. 1966).

Los resultados de resistencia a la tracción perpendicular al plano se presentan en el Cuadro $\mathrm{N}^{\circ} 5$.

\section{Cuadro $\mathrm{N}^{\circ} 5$}

TRACCIÓN PERPENDICULAR (N/mm²)

\begin{tabular}{|c|cccc|cccc|}
\hline $\begin{array}{c}\text { Aserrin } \\
(\%)\end{array}$ & Media & Mensidad $570 \mathrm{~kg} / \mathrm{m}^{3}$ & \multicolumn{4}{c|}{ Densidad $660 \mathrm{~kg} / \mathrm{m}^{3}$} \\
\hline 0 & 0.719 & 0.894 & 0.612 & 0.076 & 0.897 & 1,079 & 0,734 & 0,105 \\
20 & 0.562 & 0.757 & 0,337 & 0.114 & 1,031 & 1,165 & 0,879 & 0,087 \\
40 & 0.714 & 1.247 & 0.498 & 0.134 & 1,059 & 1,279 & 0,647 & 0,169 \\
60 & 0.902 & 1.220 & 0.600 & 0.120 & 1.118 & 1.436 & 0,828 & 0,141 \\
80 & 0.873 & 1,208 & 0,161 & 0.203 & 1,192 & 1.432 & 0,957 & 0,127 \\
100 & 0.936 & 1.157 & 0.632 & 0.121 & 1.358 & 1.577 & 1.020 & 0,158 \\
\hline
\end{tabular}

D.E.: Desviación estándar

La norma DIN establece un valor mínimo de $0.35 \mathrm{~N} / \mathrm{mm}^{2}$, valor que fue superado por los promedios de todos los tratamientos.

Al realizar el análisis de varianza entre tratamientos con un nivel de significación del $5 \%$. se comprobó que existen diferencias significativas entre los mismos.

Al probar modelos de regresión. con aserrín y densidad como variables independientes, se determinó que ambas aportan al coeficiente de regresión. aunque el efecto de la densidad es mayor. La variable densidad aporta $80 \%$ del valor de $\mathrm{r}$ y $\mathrm{r}^{2}$.

El modelo que mejor ajustó en este caso fue el siguiente:

$$
\begin{aligned}
R_{t} & =-1,608+0,00+A s+0,00+D e n \\
r & =0,81 \\
r^{2} & =0,66
\end{aligned}
$$

Donde:

$R_{t}=$ Resistencia a la tracción $\left(\mathrm{N} / \mathrm{mm}^{2}\right)$

As $=$ Contenido de aserrin $(\%)$

Den = Densidad de los tableros en $\left(\mathrm{kg} / \mathrm{m}^{3}\right)$ 
La dependencia de la resistencia a la tracción de la densidad y del contenido de aserrin es similar a la encontrada por otros autores (Brumbaugh. 1960; Gatchell et al, 1966; Poblete, 1979).

Flexión Estática (MOR y MOE)

Los reșultados obtenidos para los distintos tratamientos en módulo de ruptura se presentan en el Cuadro $\mathrm{N}^{\circ} 6$.

Al realizar un análisis de varianza. con un nivel de significación del $5 \%$, se comprobó que en ambas propiedades existen diferencias significativas entre los tratamientos.

En el caso del módulo de ruptura el análisis paso a paso comprobó que el contenido de aserrín es la variable predictora de mayor importancia aportando aproximadamente un $80 \%$ de "r".

\section{Cuadro $\mathrm{N}^{\circ} 6$ \\ FLEXION (N/mm²) (MOR Y MOE)}

\begin{tabular}{|c|c|c|c|c|c|c|c|c|c|}
\hline \multirow[t]{2}{*}{ Propiedad } & \multirow{2}{*}{$\begin{array}{c}\text { Aserrin } \\
(\%)\end{array}$} & \multicolumn{4}{|c|}{ Densidad $570 \mathrm{~kg}^{3} \mathrm{~m}^{3}$} & \multicolumn{4}{|c|}{ Densidad $660 \mathrm{~kg} / \mathrm{m}^{3}$} \\
\hline & & Media & Maxima & Minima & D. E. & Media & Máxima & Minima & D. E. \\
\hline \multirow{6}{*}{ MOR } & 0 & 17,595 & 22,650 & $14 . .630$ & 2.583 & 23,059 & 27,910 & 17,950 & 2,972 \\
\hline & 20 & 17,424 & 24.970 & 12.690 & 3.379 & 23,459 & 27.100 & 16,570 & 3.037 \\
\hline & 40 & 14,043 & 17.070 & 9.520 & 2.087 & 19.759 & 23,180 & 16.260 & 2,384 \\
\hline & 60 & 13,275 & 19.520 & 9.340 & 2.844 & 19,031 & 24.060 & 13.850 & 3,161 \\
\hline & 80 & 11,613 & 15.130 & 6.330 & 2.156 & 16,551 & 21.930 & 12,340 & 2,479 \\
\hline & 100 & 9,504 & 11.680 & 7.090 & 1.375 & 13,704 & 17,140 & 11,320 & 1,469 \\
\hline \multirow{6}{*}{ MOE } & 0 & 1.885 & 2.301 & 1.620 & 221 & 2.426 & 2.760 & 1.836 & 258 \\
\hline & 20 & 2.066 & 2.695 & 1.516 & 353 & 2.474 & 2.780 & 1.920 & 241 \\
\hline & 40 & 1.686 & 2.090 & 1.356 & 197 & 2.380 & 2.701 & 1.970 & 236 \\
\hline & 60 & 1.605 & 2.371 & 1.204 & 314 & 2.295 & 2.701 & 1.906 & 282 \\
\hline & 80 & $1.4+5$ & 1.822 & 819 & 249 & 2.070 & 2.472 & 1.607 & 241 \\
\hline & 100 & 1.198 & 1.514 & 879 & 185 & 1.626 & 2.095 & 1.290 & 213 \\
\hline
\end{tabular}

D.E.: Desviación estandar

La ecuación de la regresión para el módulo de ruptura (MOR) fue la siguiente:

$$
\begin{aligned}
M O R & =-17,4+5-0,091 \mathrm{As}+0,063 \mathrm{Den} \\
r & =0,8+5 \\
r^{2} & =0,713
\end{aligned}
$$


Donde:

MOR = Módulo de nuptura $\left(\mathrm{N} / \mathrm{mm}^{2}\right)$

As $=$ Aserrin $(\%)$

Den $=\operatorname{Densidad}\left(\mathrm{kg} / \mathrm{m}^{3}\right)$

Los valores de módulo de ruptura dieron, como se esperaba, una alta correlación con ambas variables estudiadas.

En el caso del módulo de elasticidad al probar distintos modelos de regresión se obtuvo el siguiente modelo:

$$
\begin{aligned}
\text { MOE } & =-1.576,613-7,984 \text { As }+3,816 \text { Den } \\
r & =0,814 \\
r^{2} & =0,663
\end{aligned}
$$

Donde:

$$
\begin{aligned}
& M O E=\text { Módulo de elasticidad }\left(\mathrm{N} / \mathrm{mm}^{2}\right) \\
& A s \quad=\text { Aserrin }(\%) \\
& \text { Den }=\text { Densidad }\left(\mathrm{kg} / \mathrm{m}^{3}\right)
\end{aligned}
$$

El análisis estadístico de los valores del módulo de elasticidad comprobó que la densidad del tablero es la variable predictora de mayor importancia aportando un valor de 0.599 de " $r$ ", mientras que el porcentaje de aserrín aportó el 0,215 restante.

En el caso de la resistencia a la flexión, tanto en su módulo de ruptura como de elasticidad. se trata de una propiedad que refleja el estado de calidad de las capas superficiales. En este sentido resulta especialmente importante, ya que en el presente estudio se incorporó aserrín en las capas externas.

La norma establece como exigencia una resistencia a la flexión (MOR) mínima de $16 \mathrm{~N} / \mathrm{mm}^{2}$. Con esta restricción los tratamientos de densidad de $570 \mathrm{~kg} / \mathrm{m}^{3}$ admiten hasta un $20 \%$ de aserrín. mientras que aquellos con densidades de $655 \mathrm{~kg} / \mathrm{m}^{3}$ admiten hasta un $80 \%$ de aserrín.

El módulo de elasticidad exigido por DIN 68763 debe estar entre 1900 y 2800 $\mathrm{N} / \mathrm{mm}^{2}$, lo que exigiría que los tableros sean fabricados con densidades mayores a 570 $\mathrm{kg} / \mathrm{m}^{3}$ y con contenidos máximos de aserrín de $80 \%$. 
Las resistencias obtenidas fueron iguales o mayores a las registradas por otros autores (Klauditz y Buro, 1962; Poblete, 1979). Es posible que ello se deba a que se contempló una exhaustiva clasificación de las particulas. a que los tableros fueron confeccionados de tres capas, y a que hubo una asignación de adhesivo mayor para las capas externas.

El contenido de aserrín actuó negativamente con respecto a esta propiedad mecánica. La reducción de esta propiedad se debe a las características geométricas de las partículas de aserrín. Estas presentaban una mayor superficie especifica y por lo tanto quedó una menor cantidad de adhesivo disponible para el encolado. Debe tenerse presente que además de lo anterior parte del aserrín adoptó una posición vertical, con las fibras perpendiculares a la superficie del tablero, lo que redujo la resistencia.

Es interesante destacar que esta es la única propiedad en que el aserrín tuvo mayor importancia que la densidad como variable predictora. Esto tiene importancia ya que aún aumentando la densidad del tablero. el agregar aserrin va a generar tableros con una resistencia a la flexión estática menor.

\section{Dureza Janka}

La determinación de esta propiedad resulta especialmente importante ya que no existen antecedentes de otros trabajos que la incluyan. Esta propiedad. medida normalmente en madera sólida. debicra verse afectada por la densidad de las zonas mas superficiales del material en ensayo, y refleja en parte el estado de calidad de las capas externas del tablero.

El análisis de varianza, para un nivel de significación del $5 \%$. demostró que existen diferencias significativas entre los tratamientos.

Los resultados de Dureza Janka para los diferentes tratamientos se presentan a continuación en el Cuadro $\mathrm{N}^{\circ} 7$.

Cuadro $\mathrm{N}^{0} 7$.

DIREZA JANKA (N)

\begin{tabular}{|c|c|c|c|c|c|c|c|c|}
\hline \multirow{2}{*}{$\begin{array}{c}\text { Aserrin } \\
(\%)\end{array}$} & \multicolumn{4}{|c|}{ Densidad $570 \mathrm{~kg} / \mathrm{m}^{3}$} & \multicolumn{4}{|c|}{ Densidad $660 \mathrm{~kg} / \mathrm{m}^{3}$} \\
\hline & Media & $\begin{array}{c}\text { Máxim } \\
\mathrm{a}\end{array}$ & Minima & D. E. & Media & Maxima & Minima & D. E. \\
\hline 0 & 2.825 .6 & 3.515 .7 & 2.211 .4 & 365.8 & $3.995,4$ & 4.339 .4 & $3.040,1$ & 389.1 \\
\hline 20 & $2.363,8$ & 3.339 .2 & 1.618 .1 & 502.0 & 3.643 .6 & 4.496 .3 & 2.706 .6 & 483,2 \\
\hline 40 & $2.741,4$ & 3.295 .0 & 2.088 .8 & 397.0 & $3.658,3$ & 4.437 .5 & $3.005,7$ & 463,6 \\
\hline 60 & $2.487,6$ & 3.192 .1 & 1.539 .6 & 449.0 & $3.578,6$ & $4.187,4$ & 2.853 .7 & 470,8 \\
\hline 80 & $2.656,8$ & 4.712 .1 & 1.657 .3 & 732,8 & 3.550 .4 & $4.177,6$ & $2.716,4$ & 490,9 \\
\hline 100 & $2.338,1$ & 2.814 .5 & 1.887 .8 & 315.3 & $3.161,0$ & 3.711 .8 & 2.549 .7 & 366,9 \\
\hline
\end{tabular}

D.E.: Desviación estandar 
Al probar el ajuste de los valores a un modelo de regresión se determinó la siguiente ecuación:

$$
\begin{aligned}
\text { D.J. } & =-3.265,668-4,164 A S+11,017 \text { Den } \\
r & =0,995 \\
r^{2} & =0,912
\end{aligned}
$$

Donde:

D.J. = Dureza Janka $(\mathrm{N})$

$A s=$ Aserrin $(\%)$

Den $=$ Densidad $\left(\mathrm{kg} / \mathrm{m}^{3}\right)$

Los análisis estadisticos determinaron que la densidad aportó a " $r$ " 0,934 , mientras que el aserrín aportó el 0.021 restante.

Los resultados del ensayo demostraron que esta propiedad está altamente correlacionada con el contenido de aserrín y con la densidad de los tableros. En general a mayor cantidad de aserrin menor dureza y a mayor densidad mayor dureza. Un aumento de la densidad, de $570 \mathrm{~kg} / \mathrm{m}^{3}$ a $660 \mathrm{~kg} / \mathrm{m}^{3}$, aumentó la dureza entre $34 \%$ y $54 \%$. El cfecto del aserrín no fue claro con un nivel de densidad de $570 \mathrm{~kg} / \mathrm{m}^{3}$. Con una densidad de $660 \mathrm{~kg} / \mathrm{m}^{3}$ el aumento del contenido de aserrín de 0 a $100 \%$ provocó una disminución de la dureza de un $21 \%$.

La explicación para la pérdida de dureza por la inclusión de aserrín puede encontrarse en el mayor contenido de corteza presente en esta fracción y la menor efectividad del adhesivo por la mavor superficic específica de este material.

Esta propiedad afecta a una seric de usos finales que pueden darse al tablero. En términos generales la dureza es un indicador de la calidad del tablero para efectuar recubrimientos superficiales.

Flcxión Dinámica (Impacto)

Los resultados del ensayo de Flcxión Dinámica (Impacto) se presentan en el Cuadro $\mathrm{N}^{\circ} 8$. 


\section{Cuadro $\mathrm{N}^{\circ} 8$ : \\ FLEXIÓN DINÁMICA, IMPACTO (kJoule/m²)}

\begin{tabular}{|c|c|c|c|c|c|c|c|c|}
\hline \multirow{2}{*}{$\begin{array}{c}\text { Aserrin } \\
(\%)\end{array}$} & \multicolumn{4}{|c|}{ Densidad $570 \mathrm{~kg} / \mathrm{m}^{3}$} & \multicolumn{4}{|c|}{ Densidad $660 \mathrm{~kg} / \mathrm{m}^{3}$} \\
\hline & Media & $\begin{array}{c}\text { Máxim } \\
\mathbf{a}\end{array}$ & Minima & D. E. & Media & Máxima & Minima & D. E. \\
\hline 0 & 7,585 & 9,10 & 5,14 & 1,490 & 9,596 & 12,10 & 8,10 & 1,407 \\
\hline 20 & 6,940 & 9,19 & 5,92 & 1,173 & $\mathbf{9 , 0 8 3}$ & 12,17 & $\mathbf{9 , 9 7}$ & 1,468 \\
\hline 40 & 5,715 & 7,08 & 5.06 & 0,745 & 7,261 & 8,20 & 6,07 & 0,838 \\
\hline 60 & 4,428 & 5,09 & 4,02 & 0,530 & $\mathbf{5 , 9 5 9}$ & 7,10 & 4,05 & 1,006 \\
\hline 80 & 3,515 & 4,07 & 2,98 & 0,559 & 4,814 & 6,09 & 4,02 & 0,719 \\
\hline 100 & 2,986 & 3,03 & 2,92 & 0,034 & 4,136 & 4,90 & 2,90 & 0,689 \\
\hline
\end{tabular}

D.E.: Desviación estándar

El análisis de varianza por tratamiento, para un nivel de significación del $5 \%$, demostró que existen diferencias significativas.

El modelo de regresión que mejor se ajustó a las variables estudiadas es:

$$
\begin{aligned}
& R_{i}=2,911-0,055 A S+0,01+\text { Den } \\
& r=0,902 \\
& r^{2}=0,814
\end{aligned}
$$

Donde:

$R_{l} \quad=$ Resistencia al impacto $\left(\mathrm{kJoule} / \mathrm{m}^{2}\right)$

$A s=$ Aserrin $(\%)$

Den $=$ Densidad $\left(\mathrm{kg} / \mathrm{m}^{3}\right)$

Al realizar el estudio paso a paso se determinó que la densidad aportó al valor de " $r$ " 0,827 , mientras que el aserrín contribuyó con el 0.075 restante.

Los análisis estadísticos determinaron que el aserrín se correlaciona inversamente con la flexión dinámica: la densidad actúa en forma positiva.

El efecto del aserrín fue el esperado, ya que por sus características y geometría opuso una menor resistencia. Partículas más pequeñas en longitud y con una menor cantidad especifica de adhesivo fueron más débiles ante el efecto del impacto. Al mismo tiempo, el aumentar la densidad se tradujo en una mayor resistencia al golpe del péndulo.

La flexión dinámica no ha sido estudiada en tableros con aserrín, por lo que los resultados obtenidos en este trabajo permiten conocer la tendencia del efecto de 
incorporar particulas con coeficientes de esbeltez bajos (menores a 10) sobre esta propiedad.

Teniendo en cuenta que los tableros con $100 \%$ de aserrin presentaron una resistencia del orden del $40 \%$ del valor obtenido en tableros sin aserrín, en aquellos usos donde la se requieran valores altos de flexión dinámica se deberá evitar la inclusión de aserrin.

\section{CONCLUSIONES}

$\mathrm{El}$ análisis de $\mathrm{pH}$ y densidad de las partículas y del aserrín demostró que no existen en estos aspectos diferencias entre ambos materiales. La diferencia entre aserrín y partículas se presentó en su geometría y en el mayor contenido de corteza del primero. Por esta razón a medida que aumentó el contenido de aserrín aparecieron coloraciones más oscuras en las caras.

El análisis del perfil de densidad demostró que no existen diferencias por la inclusión de aserrin.

El efecto del aserrín sobre las propiedades fisicas fue en general poco significativo, teniendo éstas una mayor relación con la variable densidad del tablero.

El hinchamiento a 2 horas sólo se vio afectado por la variable densidad, disminuyendo con incrementos de ésta. La misma propiedad a 24 horas se vio afectada por el aserrin y la densidad. Al permanecer en agua por un tiempo prolongado se rompió la unión entre las partículas y se liberó el material comprimido, por lo que al aumentar la densidad aumentó el hinchamiento. Por los valores obtenidos, la inclusión de un hidrófobo resulta indispensable.

La absorción de agua a 2 horas sólo mostró dependencia con la variable densidad. Aumentos de la densidad provocaron una disminución de la absorción, como consecuencia de una menor cantidad de espacio poroso. Con una inmersión por 24 horas aparecieron ambas variables afectando la absorción. Mientras el aserrin actuó incrementándola. la densidad actuó disminuyendo dicha variable.

La inclusión de aserrín afectó todas las propiedades mecánicas. Estas se vieron afectadas negativamente por el agregado de este material, salvo en el caso de la propiedad de Tracción perpendicular.

La propiedad limitante para la inclusión de aserrín resultó ser la flexión estática. Estc ensayo fue el único en el que la incorporación de aserrín tuvo un efecto más importante que el aumento de la densidad. Mientras la inclusión de aserrín disminuyó esta propiedad, el aumento de densidad la incrementó. Los valores obtenidos permitieron cumplir con las exigencias de la norma DIN admitiendo hasta $20 \%$ de aserrin para el caso de densidad $570 \mathrm{~kg} / \mathrm{m}^{3}$ y hasta $80 \%$ con densidad $660 \mathrm{~kg} / \mathrm{m}^{3}$. 
La resistencia a la tracción aumentó con incrementos de la densidad y el contenido de aserrín. Todos los tableros cumplieron con las exigencias de DIN 68763

La dureza Janka y la flexión dinámica (impacto) son propiedades no determinadas en estudios anteriores. En ambos casos resultó favorable aumentar la densidad y desfavorable la inclusión de aserrin.

\section{BIBLIOGRAFIA}

Berterreche, A., 1994. Efecto de la Inclusión de Aserrin Proveniente de Sierra Huincha en Algunas Propiedades Físico-mecánicas de Tableros de Particulas Fabricados con Pinus radiata D.Don. U.Austral de Chile. Tesis para optar al título de Magister en Cs. Forestales. 80 pp.

Bhagwat, S.G., 1971. Physical and Mechanical Variations in Cottonwood and Hickory Flake Boards Made from Flakes of Three Sizes. Forest Product Journal 21 (9): 101-103.

Brumbaugh, J.I., 1960. Effect of Flakes Dimensions on Properties of Particle Boards. Forest Product Journal 10 (5):243-246.

Gatchell, C.; Heebink, B; Hefty, C., 1966. Influence of Component Variables on Properties of Particleboard for Exterior Use. Forest Products Joumal 16(4):46-59.

Gerischer, G., 1977. Structural Particleboard from Waste Materials. Holzforschung 31 (4): 129133.

Gillwald, W., 1965. Zur Problematik der Herstellung von Spanplatten aus Abfallspănen. Holztechnologie 6 (1): 27-36.

Jensen, U., 1978. Einsatz Aufbereiteter Gattersägespănen in der Deckschicht von Möbelspanplatten. Holztechnologie 19 (4): 220-223.

Jorgensen, R.N.; Odell, R.L., 1961. Dimensional Stability of Oak Flake Board as Affected by Particle Geometry and Resin Spread. Forest Product Journal 11(10): 463-466.

Klauditz, W ; Buro, A., 1962. Die Eignung von Sägespänen zur Herstellung von Holzpanplatten. Holz als Roh-und Werkstoff 20:19-26.

Kusian, R., 1966. Üer den Einfluss der Spanform und-grösse auf die Eigenschaften von Sägespäneplatten. Holztechnologie 7 (1): $34-40$

Poblete, H,. 1979. Inclusión de Aserrin en Tableros de Particulas. Universidad Austral de Chile. Facultad de Ciencias Forestales. Bosque 3 (1): 39-46.

Poblete, H., 1985. Influencia del Tamaño de Particula sobre las Propiedades Mecánicas Flexión y Tracción en Tableros de Particulas Aglomerados con Ureaformaldehido. Universidad Austral de Chile, Facultad de Ciencias Forestales. In Pinus radiata Investigación en Chile. pp. 241-256.

Poblete, H.; Sanchez, J.P., 1991. Tableros con Corteza de Pinus radiata. BOSQUE 12 (1): 17. 26.

Post, P.W., 1958. The Effect of Particle Geometry and Resin Content on Bending Strength of Oak Fake Board. Forest Product Journal 8 (10):317-322. 